\title{
Formation of quasicrystals and amorphous-to-quasicrystalline phase transformation kinetics in Zr65Al7.5Ni10Cu7.5Ag10 metallic glass under pressure
}

Jiang, Jianzhong; Zhuang, Yanxin; Rasmussen, Helge Kildahl; Saida, J.; Inoue, A.

\section{Published in:}

Physical Review B

Link to article, DOI:

10.1103/PhysRevB.64.094208

Publication date:

2001

Document Version

Publisher's PDF, also known as Version of record

Link back to DTU Orbit

Citation (APA):

Jiang, J., Zhuang, Y., Rasmussen, H. K., Saida, J., \& Inoue, A. (2001). Formation of quasicrystals and amorphous-to-quasicrystalline phase transformation kinetics in Zr65Al7.5Ni10Cu7.5Ag10 metallic glass under pressure. Physical Review B, 64(9), 094208. https://doi.org/10.1103/PhysRevB.64.094208

\section{General rights}

Copyright and moral rights for the publications made accessible in the public portal are retained by the authors and/or other copyright owners and it is a condition of accessing publications that users recognise and abide by the legal requirements associated with these rights.

- Users may download and print one copy of any publication from the public portal for the purpose of private study or research.

- You may not further distribute the material or use it for any profit-making activity or commercial gain

- You may freely distribute the URL identifying the publication in the public portal 


\title{
Formation of quasicrystals and amorphous-to-quasicrystalline phase transformation kinetics in $\mathrm{Zr}_{65} \mathrm{Al}_{7.5} \mathrm{Ni}_{10} \mathrm{Cu}_{7.5} \mathrm{Ag}_{10}$ metallic glass under pressure
}

\author{
J. Z. Jiang, * Y. X. Zhuang, and H. Rasmussen \\ Department of Physics, building 307, Technical University of Denmark, DK-2800, Lyngby, Denmark \\ J. Saida \\ Inoue Superliquid Glass Project, ERATO, Japan Science and Technology Corporation (JST), Sendai 982-0807, Japan
}

A. Inoue

Institute of Materials Research, Tohoku University, Sendai 980-8577, Japan

(Received 14 November 2000; revised manuscript received 6 June 2001; published 15 August 2001)

\begin{abstract}
The effect of pressure on the formation of quasicrystals and the amorphous-to-quasicrystalline phase transformation kinetics in the supercooled liquid region for a $\mathrm{Zr}_{65} \mathrm{Al}_{7.5} \mathrm{Ni}_{10} \mathrm{Cu}_{7.5} \mathrm{Ag}_{10}$ metallic glass have been investigated by in situ high-pressure and high-temperature nonisothermal and isothermal $\mathrm{x}$-ray powder diffraction measurements using synchrotron radiation, respectively. It is found that with increasing pressure, the onset temperature for the formation of quasicrystals increases with a slope of $9.4 \mathrm{~K} / \mathrm{GPa}$ while the temperature interval of the stability and the average grain size of quasicrystals decrease. Atomic mobility is important for the formation of quasicrystals from the metallic glass whereas the relationship of the crystallization temperature vs pressure for the transition from the quasicrystalline state to intermetallic compounds may mainly depend on the thermodynamic potential energy barrier. To study the amorphous-to-quasicrystalline phase transformation kinetics in the metallic glass, relative volume fractions of the transferred quasicrystalline phase as a function of annealing time, obtained at 663, 673, 683, and $693 \mathrm{~K}$, have been analyzed in details using 14 nucleation and growth models together with the Johnson-Mehl-Avrami model. The Avrami exponent was found to be near 1 at all four temperatures, also indicating that atomic diffusion might involve in the amorphous-toquasicrystalline phase transformation for the $\mathrm{Zr}_{65} \mathrm{Cu}_{7.5} \mathrm{Al}_{7.5} \mathrm{Ni}_{10} \mathrm{Ag}_{10}$ metallic glass. It is found that the timedependent transient nucleation is essential for the transformation and different nucleation and growth models have been critically assessed.
\end{abstract}

DOI: 10.1103/PhysRevB.64.094208

PACS number(s): 61.44.Br, 61.43.Dq, 64.70.Kb, 61.50.Ks

\section{INTRODUCTION}

In a variety of systems it has been demonstrated that icosahedral quasicrystals can be synthesized by crystallization of amorphous alloys. In 1996, Koster et al. reported the formation of icosahedral quasicrystals in a $\mathrm{Zr}-\mathrm{Al}-\mathrm{Ni}-\mathrm{Cu}$ amorphous alloy with a wide supercooled liquid region. ${ }^{1}$ Since then, there has been considerable interest in the amorphous-to-quasicrystalline phase transformation in $\mathrm{Zr}$ based alloys, ${ }^{2-17}$ such as $\mathrm{ZrM}(M=\mathrm{Pd}$ and $\mathrm{Pt}),{ }^{2,3} \mathrm{ZrNi} M($ $M=\mathrm{Pd}, \mathrm{Au}, \mathrm{Pt}$, and $\mathrm{Ti}),{ }^{4,5} \mathrm{ZrCu} M \quad(\mathrm{Al}$ and $\mathrm{Pd}),{ }^{6,7}$ $\mathrm{ZrAlCuPd},{ }^{7} \mathrm{ZrCuNiPd}^{7} \mathrm{ZrAlNi} M(M=\mathrm{Cu}, \mathrm{Pd}, \mathrm{Au}$, and $\mathrm{Pt}),{ }^{1,8-10} \mathrm{ZrAlNiCu} M(M=\mathrm{Ti}, \mathrm{Au}, \mathrm{Pt}, \mathrm{Pd}$, and $\mathrm{Ag}),{ }^{7,11-16}$ and $\mathrm{ZrTiCuNiBe},{ }^{17}$ but the transformation mechanism is not completely understood. Inoue and his co-workers ${ }^{12-15}$ found that the ability of quasicrystal formation from Zr-based amorphous alloys can be largely enhanced by adding noble elements (e.g., Ag and Pd) and bulk nanoquasicrystalline Zrbased materials prepared exhibit enhanced strength and ductility as compared with those for the corresponding amorphous alloys. They suggested a polymorphous reaction for the transformation for the Ag-containing alloys, while Lee et $a{ }^{16}{ }^{16}$ reported that formation of quasicrystal in the $\mathrm{ZrAlNiCuAg}$ system may involve in partitioning of solute. It has been demonstrated in many metallic glasses that applied pressure can change crystallization processes, e.g., enhancement of crystallization temperature where atomic diffusion process is a dominant factor for crystallization. ${ }^{18-21}$ The pressure dependence of the crystallization temperature obtained in the system might shed light to the nature of the amorphous-to-quasicrystal transformation. In this work, we report the pressure effect on the quasicrystal formation and the amorphous-to-quasicrystalline phase transformation kinetics in the supercooled liquid region for the $\mathrm{Zr}_{65} \mathrm{Al}_{7.5} \mathrm{Ni}_{10} \mathrm{Cu}_{7.5} \mathrm{Ag}_{10}$ metallic glass, obtained from in situ high-pressure and high-temperature nonisothermal and isothermal $\mathrm{x}$-ray powder diffraction measurements using synchrotron radiation, respectively.

\section{EXPERIMENT}

A ribbon sample of the $\mathrm{Zr}_{65} \mathrm{Al}_{7.5} \mathrm{Ni}_{10} \mathrm{Cu}_{7.5} \mathrm{Ag}_{10}$ metallic glass with a cross section of $0.03 \times 1 \mathrm{~mm}$ was prepared by the melt-spinning technique from a master alloy ingot prepared by arc melting in an Ar atmosphere. The amorphous nature of the as-quenched ribbon was confirmed by $\mathrm{x}$-ray powder diffraction and transmission electron microscopy. Thermal analysis was performed in a differential scanning calorimeter (DSC) at a heating rate of $40 \mathrm{~K} / \mathrm{min}$ under a flow of purified argon. The alloy exhibits an endothermic event characteristic of the glass transition, followed by two characteristic exothermic events indicating a two-stage phase transformation process. It was found that the glass transition temperature $\left(T_{g}\right)$ and the onset temperatures of the first and 

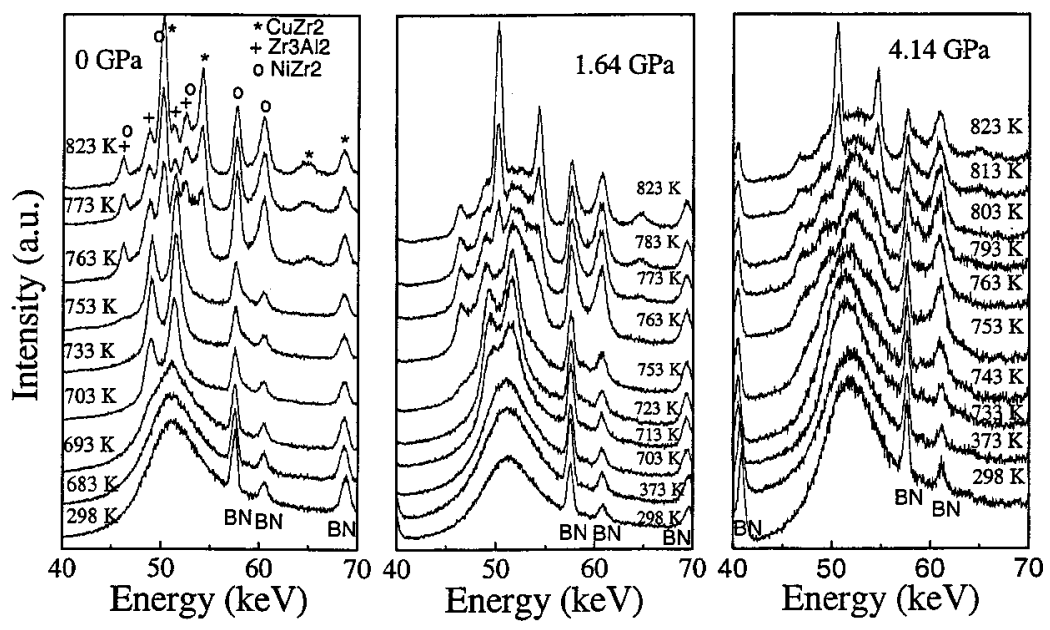

FIG. 1. In situ energy dispersive $\mathrm{x}$ ray powder diffraction patterns recorded for the $\mathrm{Zr}_{65} \mathrm{Al}_{7.5} \mathrm{Ni}_{10} \mathrm{Cu}_{7.5} \mathrm{Ag}_{10}$ metallic glass at $0 \quad \mathrm{GPa} \quad(E d=124.669 \mathrm{keV} \AA), \quad 1.64 \quad \mathrm{GPa}$ $(E d=124.677 \mathrm{keV} \AA)$, and $4.14 \quad \mathrm{GPa} \quad(E d$ $=124.592 \mathrm{keV} \AA$ ) and various temperatures. second crystallization events $\left(T_{x 1}\right.$ and $\left.T_{x 2}\right)$ are 657,706 , and $763 \mathrm{~K}$, respectively.

In situ high-pressure and high-temperature nonisothermal and isothermal energy-dispersive x-ray powder diffraction (EDXRD) measurements were performed using synchrotron radiation at the MAX80 station, HASYLAB in Hamburg, Germany. ${ }^{22}$ The cubic sample assembly is compressed by six truncation anvils of tungsten carbide in a 250-ton hydraulic press. Electric current is sent through a graphite heater via two appropriate anvils. The temperature is measured by means of a thermocouple voltage with a stability of $\pm 1 \mathrm{~K}$. Each nonisothermal run consists of an isothermal roomtemperature compression followed by an isobaric heating to high temperature in steps of $10 \mathrm{~K}$. The average heating rate in the temperature range from 298 to $873 \mathrm{~K}$ was roughly estimated to be $3 \mathrm{~K} / \mathrm{min}$. Each isothermal kinetic run consists of an isothermal room-temperature compression to $0.86 \mathrm{GPa}$ followed by an isobaric heating to a given temperature rapidly and an isothermal annealing. The heating rate is estimated to be around $40 \mathrm{~K} / \mathrm{min}$. The EDXRD patterns were automatically recorded every $5 \mathrm{~min}$. The pressure of the sample is calculated from the lattice constant of $\mathrm{NaCl}$ using the Decker equation of state. ${ }^{23}$ Pure $\mathrm{Zr}, \mathrm{Fe}$, and the metallic glass were used to examine the possible oxidation of samples during the heat treatments using the sample assembly. It was found that only pure metallic phases in the three systems were detected after heat treatments at temperatures up to $873 \mathrm{~K}$.

\section{RESULTS AND DISCUSSION}

\section{A. Nonisothermal measurements}

In situ high-temperature EDXRD measurements of the $\mathrm{Zr}_{65} \mathrm{Al}_{7.5} \mathrm{Ni}_{10} \mathrm{Cu}_{7.5} \mathrm{Ag}_{10}$ metallic glass were performed in a pressure range of $0-4.2 \mathrm{GPa}$. EDXRD patterns were recorded every $10 \mathrm{~K}$ in order to observe the onset temperature of crystallization within an uncertainty of $10 \mathrm{~K}$. The crystalline phases determined from the EDXRD patterns recorded are identical in the pressure range used. Figure 1 exemplifies EDXRD patterns recorded for the sample at 0, 1.64, and 4.14 $\mathrm{GPa}$ and various temperatures. A broad amorphous peak, located at $E \approx 51 \mathrm{keV}$, together with a few Bragg peaks from
$\mathrm{BN}$ are observed in the EDXRD patterns recorded at $298 \mathrm{~K}$ and three pressures. At $P=0 \mathrm{GPa}$, the EDXRD pattern recorded at $683 \mathrm{~K}$ is similar with that at $298 \mathrm{~K}$ while change in shape is detected in the pattern recorded at $693 \mathrm{~K}$. At $703 \mathrm{~K}$, two new Bragg peaks at $E \approx 49$ and $51.5 \mathrm{keV}$ appear and can be indexed to (100000) and (110000) peaks for a primitive icosahedral structure. ${ }^{17}$ The third strongest peak (101000) of the primitive icosahedral phase locates at approximately 82.2 $\mathrm{keV}$, above the range that we measured. For clarification of the formation of quasicrystal in the annealed samples, conventional XRD measurements of one sample annealed at 0 $\mathrm{GPa}$ and $673 \mathrm{~K}$ for $60 \mathrm{~min}$ using a $\mathrm{Cu} K \alpha$ radiation was performed as shown in Fig. 2. A primitive icosahedral structure was indeed found to be the most promising indexing scheme. No intermetallic crystalline compounds are detected, indicating that only an amorphous-to-quasicrystal transition occurs at around $T_{x 1}=698 \pm 10 \mathrm{~K}$ in the metallic glass at ambient pressure. With further increasing temperature, linewidths of the Bragg peaks for the quasicrystal remain almost unchanged up to $753 \mathrm{~K}$, at which a tiny new peak at $E \approx 46 \mathrm{keV}$ appears. At $763 \mathrm{~K}$, more new Bragg peaks are observed and they remain in position up to temperatures at $823 \mathrm{~K}$ while the relative intensities vary with temperature. These new peaks can be attributed to three intermetallic compounds: $\mathrm{CuZr}_{2}$-like, $\mathrm{NiZr}_{2}$-like, and

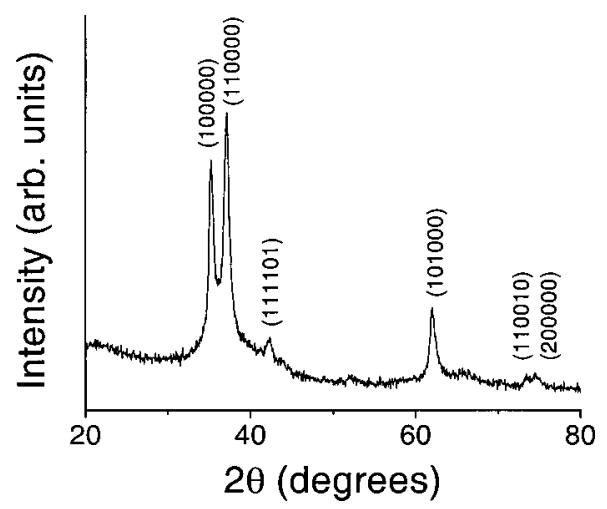

FIG. 2. The $\mathrm{x}$-ray powder diffraction pattern recorded for the $\mathrm{Zr}_{65} \mathrm{Al}_{7.5} \mathrm{Ni}_{10} \mathrm{Cu}_{7.5} \mathrm{Ag}_{10}$ metallic glass annealed at $0 \mathrm{GPa}$ and $673 \mathrm{~K}$ for 60 min using a $\mathrm{Cu} K \alpha$ radiation. 


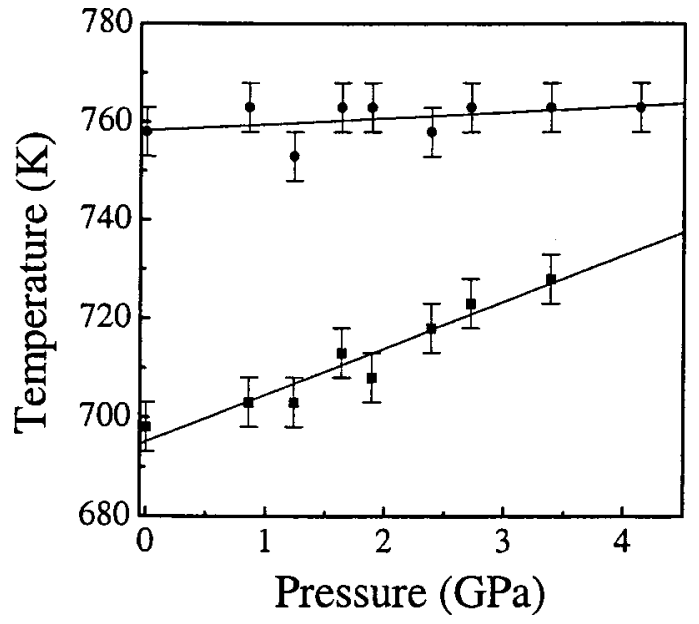

FIG. 3. Crystallization temperatures of the $\mathrm{Zr}_{65} \mathrm{Al}_{7.5} \mathrm{Ni}_{10} \mathrm{Cu}_{7.5} \mathrm{Ag}_{10}$ metallic glass as a function of pressure. The data were linearly fitted as solid lines.

$\mathrm{Zr}_{2} \mathrm{Al}_{3}$-like phases. These results reveal that the quasicrystals formed are metastable $(698-753 \mathrm{~K})$ and decompose into three intermetallic compounds at around $T_{x 2}=758 \mathrm{~K}$. With increasing pressure, the two-stage crystallization process is also observed as shown in Fig. 1 at $1.64 \mathrm{GPa}$. Figure 3 shows both crystallization temperatures as a function of pressure. It is clear that $T_{x 1}$ increases with pressure having a slope of 9.4 $\mathrm{K} / \mathrm{GPa}$ while $T_{x 2}$ remains almost unchanged in the pressure range used. At $4.14 \mathrm{GPa}$, due to broadened linewidths and a small difference between $T_{x 1}$ and $T_{x 2}$, it is difficult to estimate the value of $T_{x 1}$. One striking feature observed from the EDXRD patterns in Fig. 4 is that the linewidths of the quasicrystals formed increase with pressure. The higher the pressure, the smaller the average grain size (and/or the higher degree the disorder) of the quasicrystal, indicating that the growth of quasicrystals is suppressed under pressure. The same is true of the intermetallic compounds.

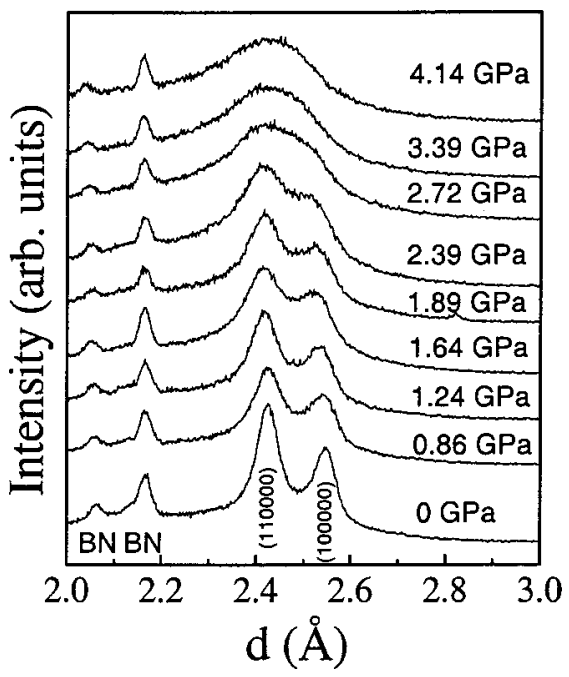

FIG. 4. In situ energy dispersive $\mathrm{x}$-ray powder diffraction patterns recorded for the $\mathrm{Zr}_{65} \mathrm{Al}_{7.5} \mathrm{Ni}_{10} \mathrm{Cu}_{7.5} \mathrm{Ag}_{10}$ metallic glass at 723 $\mathrm{K}$ under various pressures.
Crystallization of a metallic glass is normally regarded as a process proceeding by nucleation and subsequent growth of crystals. The onset crystallization temperature of an $A$-to- $B$ phase transformation may be governed by the thermodynamic potential energy barrier of nucleation and diffusion activation energy. According to crystallization kinetics theory, the nucleation rate $I$ can be written as $I=I_{0} / \exp \left[\left(\Delta G^{*}+Q_{n}\right) / k_{B} T\right]$, where $I_{0}$ is a constant, $\Delta G^{*}$ is the free energy required to form a nucleus of the critical size, i.e., the thermodynamic potential energy barrier of nucleation, $Q_{n}$ is the activation energy for the transport of an atom across the interface of an embryo, and $k_{B}$ is the Boltzmann's constant. For the first crystallization reaction, from amorphous-to-quasicrystal, the interfacial energy of quasicrystals is usually small, e.g., $\sigma \sim 13 \mathrm{~mJ} / \mathrm{m}^{2}$ for $\mathrm{Zr}_{69.5} \mathrm{Al}_{7.5} \mathrm{Ni}_{11} \mathrm{Cu}_{12}$ alloy and $\sigma \sim 2-15 \mathrm{~mJ} / \mathrm{m}^{2}$ for $\mathrm{Al}_{75} \mathrm{Cu}_{15} \mathrm{~V}_{10}$ alloy ${ }^{24} \Delta G^{*}$, which is proportional to $\sigma^{3}$ and $1 /(P \Delta V+\Delta G)^{2}$, could be negligible, where $\Delta V$ and $\Delta G$ are the changes of molar volume and free energy between $A$ and $B$ phases. Thus the nucleation rate of the formation of quasicrystals mainly depends on $Q_{n}$, which is usually enhanced with pressure. Consequently, the enhancement of $T_{x 1}$ with pressure observed here indicates that the atomic mobility is involved in the formation and the growth of quasicrystals from the $\mathrm{Zr}_{65} \mathrm{Al}_{7.5} \mathrm{Ni}_{10} \mathrm{Cu}_{7.5} \mathrm{Ag}_{10}$ metallic glass. Isothermal kinetic measurements could gain further information of the amorphous-to-quasicrystalline phase transformation reported in the literature, ${ }^{24,25}$ which will be discussed in the next section. For the relationship of $T_{x 2}$ vs $P$, it could be tentatively explained as follows. For the second reaction, from quasicrystalline-to-intermetallic crystalline compounds, the interfacial energies for intermetallic $\mathrm{Zr}_{2} M(M=\mathrm{Cu}$ and $\mathrm{Ni})$ compounds are large ${ }^{26}$ and $\Delta G^{*}$, rather than $Q_{n}$, could become the deciding factor for the nucleation rate. If the term $P \Delta V$ is much smaller than $\Delta G, \Delta G^{*}$ is then insensitive to pressure. This implies that $T_{x 2}$ is almost constant with respect to pressure although for the second reaction atomic rearrangements in a larger scale than for the first reaction may be required. This is the case for the second crystallization reaction in the $\mathrm{Zr}_{65} \mathrm{Al}_{7.5} \mathrm{Ni}_{10} \mathrm{Cu}_{7.5} \mathrm{Ag}_{10}$ metallic glass.

\section{B. Isothermal measurements}

To monitor the amorphous-to-quasicrystalline phase transformation kinetics of the $\mathrm{Zr}_{65} \mathrm{Al}_{7.5} \mathrm{Ni}_{10} \mathrm{Cu}_{7.5} \mathrm{Ag}_{10}$ metallic glass, in situ isothermal EDXRD measurements were carried out at pressure of $0.86 \mathrm{GPa}$ and temperatures of $663 \mathrm{~K}$ for $596 \mathrm{~min}, 673 \mathrm{~K}$ for $627 \mathrm{~min}, 683 \mathrm{~K}$ for $376 \mathrm{~min}, 693 \mathrm{~K}$ for $214 \mathrm{~min}$ and $698 \mathrm{~K}$ for $7.5 \mathrm{~min}$. The quasicrystalline phase determined from EDXRD patterns is identical at all temperatures. At $698 \mathrm{~K}$, the quasicrystalline phase was detected after just $2 \mathrm{~min}$, at which the kinetic is faster than the experimental setup here. At other four temperatures, the kinetic process becomes slower and can be clearly revealed from EDXRD measurements. Figure 5 exemplifies EDXRD patterns recorded for the sample at $673 \mathrm{~K}$ for various annealing times. A broad amorphous peak, located at $E \approx 51 \mathrm{keV}$ together with a few Bragg peaks from BN, are observed in EDXRD patterns recorded at the beginning, while a change in shape 


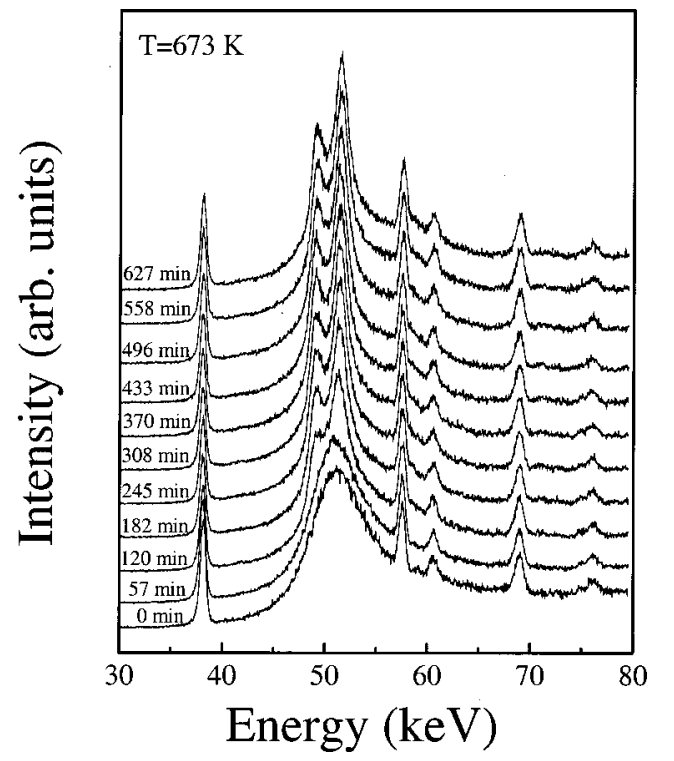

FIG. 5. In situ energy dispersive $\mathrm{x}$-ray powder diffraction patterns recorded at pressure of $0.86 \mathrm{GPa}$ and $673 \mathrm{~K}$ as a function of time for the $\mathrm{Zr}_{65} \mathrm{Cu}_{7.5} \mathrm{Al}_{7.5} \mathrm{Ni}_{10} \mathrm{Ag}_{10}$ metallic glass. $E d$ $=124.507 \mathrm{keV} \AA$.

for the amorphous peak is detected in the pattern recorded for $57 \mathrm{~min}$. With increasing time, the quasicrystalline peaks increase in intensity, indicating the volume fraction of the quasicrystalline phase increases. No intermetallic compounds were detected from EDXRD patterns recorded during the measurements, meaning that only an amorphous-toquasicrystalline phase transition occurs in the temperature and annealing time ranges used here.

In order to determine the quasicrystalline volume fraction as a function of time, EDXRD patterns recorded were fitted under the assumption that each EDXRD pattern is described as a linear combination of the EDXRD patterns $I(0)$ and $I(t$ max) recorded at $t=0$ and $t$ max, respectively. Thus the relative volume fraction $f(t)$ of the quasicrystalline phase at a given time $t$ is estimated from the expression ${ }^{27}$

$$
I(t)=[1-f(t)] I(0)+f(t) I(t \max ),
$$

where $I(t)$ is the EDXRD pattern at time $t$. The assumption made here is not unreasonable since the scattering intensity of the quasicrystalline phase is proportional to the volume of the phase. Figure 6 exemplifies an EDXRD pattern recorded at $673 \mathrm{~K}$ for $333 \mathrm{~min}$, together with the fitting curve and patterns recorded at $t=0$ and $t=627 \mathrm{~min}$. Figure 7 shows the relative volume fractions estimated from Eq. (1) as a function of time at four annealing temperatures. The shape of the $f(t)$ curves is typical " $\mathrm{S}$ " type.

Usually the fraction of the quasicrystalline component as a function of time is calculated in order to determine the kinetic law of the amorphous-to-quasicrystalline phase transformation. In this section, we discuss several models reported to analyze the experimental kinetic data obtained here. In many cases, the time evolution of the fraction of a phase is often represented by a phenomenological model describing the kinetics of isothermal phase transformation,

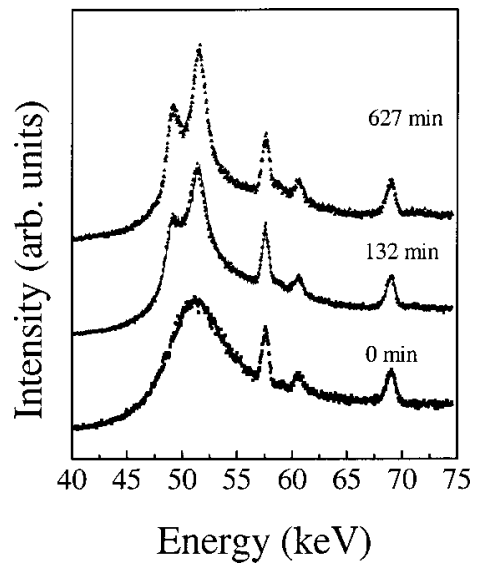

FIG. 6. In situ energy dispersive x-ray powder diffraction (EDXRD) patterns (dotted lines) recorded at $673 \mathrm{~K}$ for 0,333 , and 627 min together with the fitting curve (solid line) using Eq. (1) for the EDXRD pattern recorded for 333 min. $E d=124.507 \mathrm{keV} \AA$.

known as the Johnson-Mehl-Avrami (JMA) model. ${ }^{28-30}$ The essence of the model can be written as a very simple fomula commonly referred to as the JMA equation:

$$
x(t)=1-\exp \left\{-[k(t-\tau)]^{n}\right\},
$$

where $x(t)$ is the volume fraction of the transformed phase, $t$ the annealing time, $n$ a constant related to the dimensionality of nucleation and growth, $k$ a kinetic constant of the process which depends on temperature and effective activation energy $E_{a}$ by $k=k_{0} \exp \left(-E_{a} / R T\right)$, where $k_{0}$ is a constant and $R$ is the gas constant, and $\tau$ the incubation time which can be expressed as $\tau=\tau_{0} \exp \left(E_{a} / R T\right)$, where $\tau_{0}$ is a constant. Equation (2) can fit the experimental results well, as shown in Fig. 7 by solid curves, for annealing times longer than $\tau$. The values of $k, \tau$, and $n$ obtained from the fitting are listed in Table I. Both $k$ and $n$ are good experimental parameters for kinetic studies and are usually estimated from the inter-

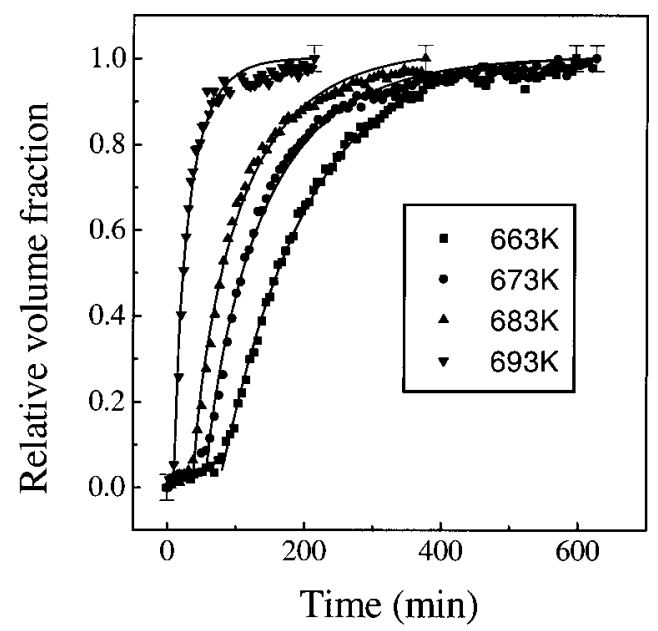

FIG. 7. Relative volume fractions of the quasicrystalline phase as a function of time during isothermal annealing treatments at pressure of $0.86 \mathrm{GPa}$ and four temperatures for the $\mathrm{Zr}_{65} \mathrm{Cu}_{7.5} \mathrm{Al}_{7.5} \mathrm{Ni}_{10} \mathrm{Ag}_{10}$ metallic glass. The solid lines are the fitting curves by the JMA equation. 
TABLE I. Values of $k, \tau$, and $n$ were deduced from the JMA equation at four temperatures.

\begin{tabular}{clcccc}
\hline \hline \multicolumn{2}{c}{ Parameters } & $663 \mathrm{~K}$ & $673 \mathrm{~K}$ & $683 \mathrm{~K}$ & $693 \mathrm{~K}$ \\
\hline \multirow{2}{*}{ From Fig. 7} & $\tau$ min & $75 \pm 3$ & $55 \pm 3$ & $37 \pm 3$ & $10 \pm 3$ \\
& $N$ & $0.0081 \pm 0.0005$ & $0.012 \pm 0.0005$ & $0.015 \pm 0.0005$ & $0.049 \pm 0.002$ \\
& $k\left(\mathrm{~min}^{-1}\right)$ & $1.04 \pm 0.05$ & $0.85 \pm 0.05$ & $0.82 \pm 0.05$ & $0.79 \pm 0.05$ \\
From Fig. 8 & $N$ & $0.0084 \pm 0.0005$ & $0.0123 \pm 0.0005$ & $0.0159 \pm 0.0005$ & $0.0486 \pm 0.0005$ \\
& $N$ & $1.10 \pm 0.02$ & $0.99 \pm 0.02$ & $0.97 \pm 0.02$ & $1.01 \pm 0.02$ \\
\hline \hline
\end{tabular}

cept and slope, respectively, of a $\ln [-\ln (1-x)]$ versus $\ln (t$ $-\tau)$ plot for a limited experimental data, providing the value of $\tau$. Figure 8 shows the plot of $\ln [-\ln (1-x)]$ versus $\ln (t$ $-\tau)$ at four temperatures for the data of $x=0.2-0.8$, in which the values of $\tau$ obtained from Eq. (2) are used. The values of $k$ and $n$ deduced from the slopes and intercepts are also listed in Table I, which are in accordance with those obtained directly from Eq. (2). The effective activation energy for the formation of quasicrystals from the metallic glass under the pressure of $0.86 \mathrm{GPa}$ is found to be 212 \pm 40 or $247 \pm 45 \mathrm{~kJ} / \mathrm{mol}$, deduced from the plots of $\ln k$ vs $1 / T$ or $\ln \tau$ vs $1 / T$ as displayed in Fig. 9, respectively. Both methods give almost the same value for the effective activation energy within experimental uncertainty, which is much smaller than $366 \mathrm{~kJ} / \mathrm{mol}$ at the ambient pressure reported in the literature. ${ }^{14}$ The smaller the effective activation energy the lower the effective energy barrier for the nucleation and growth process. One plausible explanation for this discrepancy in activation energy might be the different techniques used to estimate the fraction data versus time, DSC in Ref. 14 and XRD for the present study. Further studies are required to clarify the discrepancy. The prefactors $k_{0}$ and $\tau_{0}$ are calculated as $6.60 \times 10^{12} \mathrm{~s}^{-1}$ and $4.98 \times 10^{-20} \mathrm{~s}$, respectively. One striking feature observed from both fitting (Figs. 7 and 8) is that the Avrami exponent $n$ is about 1, which is much smaller than 4 reported in the literature. ${ }^{12-14,16}$ The low value of $n$ obtained here indicates that atomic diffusion may involve in the amorphous-to-quasicrystalline phase transformation in the $\mathrm{Zr}_{65} \mathrm{Al}_{7.5} \mathrm{Ni}_{10} \mathrm{Cu}_{7.5} \mathrm{Ag}_{10}$ metallic glass. This is

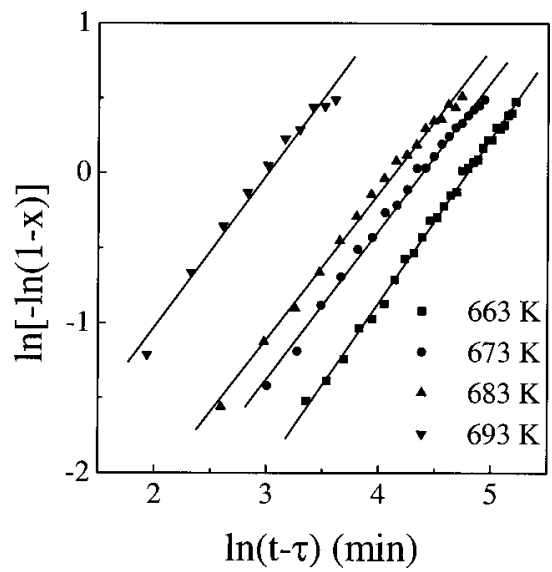

FIG. 8. JMA plots, $\ln [-\ln (1-x)]$ versus $\ln (t-\tau)$, at four temperatures for the $\mathrm{Zr}_{65} \mathrm{Cu}_{7.5} \mathrm{Al}_{7.5} \mathrm{Ni}_{10} \mathrm{Ag}_{10}$ metallic glass, in which the data for $0.2<x<0.8$ are used. consistent with the conclusion derived in Ref. 16 and from our nonisothermal XRD measurements. Further work is still required to understand the mechanism for the discrepancy in the Avrami exponent $n$.

It is clear from Fig. 7 that (i) detectable volume fractions of the quasicrystalline phase by EDXRD require incubation times at all four temperatures used here and (ii) the JMA model cannot describe the data for annealing times less than the incubation times. Relative volume fractions obtained from the fitting [Eq. (1)] in the incubation time are around $2-3 \%$, which could be due to experimental uncertainty and the contribution of quenched-in nuclei (details given later). These results infer an existence of transient nucleation process for the amorphous-to-quasicrystalline phase transformation in the $\mathrm{Zr}_{65} \mathrm{Al}_{7.5} \mathrm{Ni}_{10} \mathrm{Cu}_{7.5} \mathrm{Ag}_{10}$ metallic glass. Furthermore, it was reported that quasicrystals, formed during isothermal annealing treatments in the supercooled liquid region for the $\mathrm{Zr}_{65} \mathrm{Al}_{7.5} \mathrm{Ni}_{10} \mathrm{Cu}_{7.5} \mathrm{Ag}_{10}$ metallic alloy, rapidly grow and then saturate to approximately $30 \mathrm{~nm} .^{12-14}$ Thus we attempt to analyze the kinetic data obtained here using various nucleation and growth models. They are (i) model 1: quenched-in nucleation with three-dimensional constant growth rate; (ii) model 2: quenched-in nucleation with constant grain size $V_{o}$; (iii) model 3: steady-state nucleation $I_{\text {st }}$ with three-dimensional constant growth rate; (iv) model 4: steady-state nucleation with constant grain size; (v) model 5: quenched-in and steady-state nucleation with constant growth rate; (vi) model 6: quenched-in and steady-state

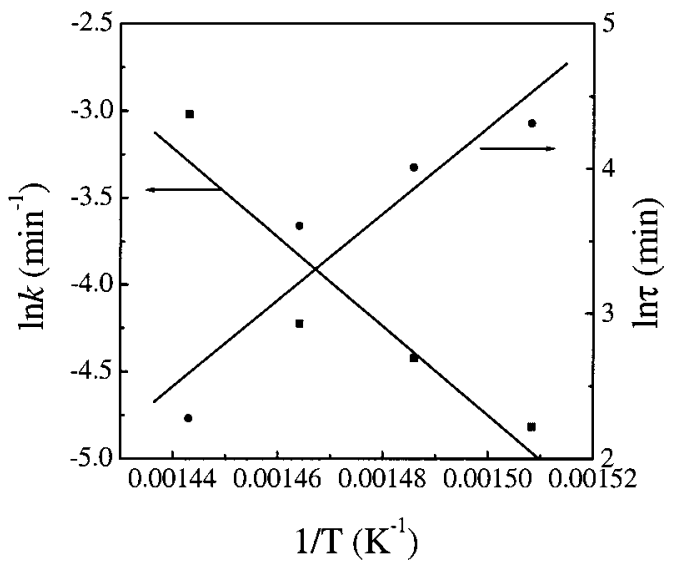

FIG. 9. Arrehnius plots of induction time $\tau$ and effective rate constant $k$ as a function of temperature for the amorphous-toquasicrystalline phase transformation in the $\mathrm{Zr}_{65} \mathrm{Cu}_{7.5} \mathrm{Al}_{7.5} \mathrm{Ni}_{10} \mathrm{Ag}_{10}$ metallic glass, in which $\tau$ and $k$, listed in Table I, are used. 
nucleation with constant grain size; (vii) model 7: timedependent Kashchiev's nucleation ${ }^{31}$ with three-dimensional constant growth rate; (viii) model 8: time-dependent Kashchiev's nucleation with constant grain size; (ix) model 9: time-dependent Zeldovich's nucleation ${ }^{32}$ with threedimensional constant growth rate; (x) model 10: timedependent Zeldovich's nucleation with constant grain size; (xi) model 11: time-dependent Kashchiev's nucleation with three-dimensional constant growth rate at $t<t_{o}$ and zero growth rate at $t>t_{o}$; (xii) model 12: time-dependent Zeldovich's nucleation with three-dimensional constant growth rate at $t<t_{o}$ and zero growth rate at $t>t_{o}$; (xiii) model 13: quenched-in and time-dependent Zeldovich's nucleation with three-dimensional constant growth rate; and (xiv) model 14: quenched-in and time-dependent Zeldovich's nucleation with constant grain size. The relative volume fraction formulas of various models are listed in the Appendix. It was found that models 1-6, cannot fit the data at all. Due to fast nucleation processes in amorphous alloys, steady-state nucleation has been considered in most cases. Very few comprehensively experimental investigations of time-dependent nucleation in metallic glasses have been reported. ${ }^{27,33}$ However, there is extensive literature on time-dependent nucleation in nonmetallic glasses, where the nucleation processes could be much slower than those in amorphous alloys. The role of this transient nucleation in devitrification of oxide glasses has been discussed in detail by Gutzow. ${ }^{34}$ In classic transient nucleation theory, two analyses, based on the ZeldovichFrenkel equation, ${ }^{32}$ were proposed. One is Zeldovich's equation. Zeldovich ${ }^{32}$ assumed that the formation work of a nucleus with size of $d$ is proportional to $d^{2}$ and the rate of monomer addition to a nucleus with size of $\mathrm{d}, k_{d}^{+} \approx k_{d^{*}}^{+}$, and found a time-dependent nucleation rate $I_{d} *(t)$ at the critical size $d^{*}$ as

$$
I_{d *}=I_{\mathrm{st}-Z} \exp \left(-\tau_{Z} / t\right)
$$

where $\tau_{Z}$ is the transient nucleation time. The other is the Kashchiev's equation. ${ }^{31}$ Kashchiev further studied the transient nucleation process and performed the most thorough analytical treatment of the Zeldovich-Frenkel equation. Based on two assumptions: (i) the formation work of a nucleus with size of $d$ is approximated by the first two nonzero terms in a Taylor expansion about $d^{*}$, and (ii) $k_{d}^{+}$ $\approx k_{d *}^{+}$, he derived the time-dependent nucleation rate as

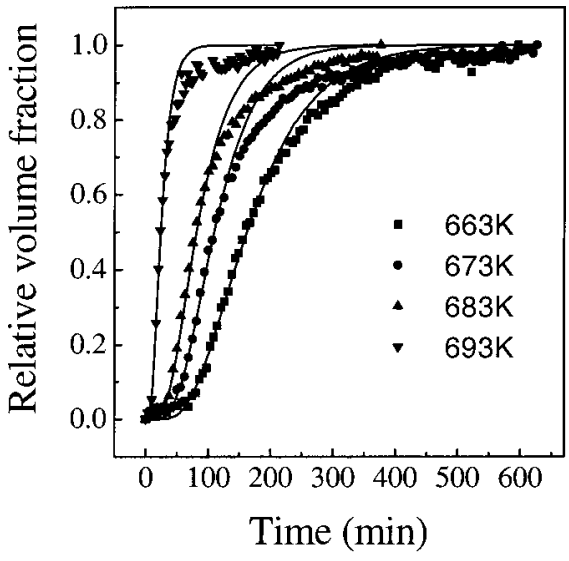

FIG. 10. Relative volume fractions of the quasicrystalline phase as a function of time during isothermal annealing treatments at pressure of $0.86 \mathrm{GPa}$ and four temperatures for the $\mathrm{Zr}_{65} \mathrm{Cu}_{7.5} \mathrm{Al}_{7.5} \mathrm{Ni}_{10} \mathrm{Ag}_{10}$ metallic glass. The solid lines are the fitting curves using model 8 (details given in text).

$$
I_{d *}=I_{\mathrm{st}-K}\left[1+2 \sum_{m=1}^{\infty}(-1)^{m} \exp \left(-\frac{m^{2} t}{\tau_{K}}\right)\right],
$$

where $\tau_{K}$ is the transient nucleation time. We found that models 8,10 , or 14 give better fit than models 7,9 , and 13 , respectively. This is consistent with experimental observation of grain growth behavior in the $\mathrm{Zr}_{65} \mathrm{Al}_{7.5} \mathrm{Ni}_{10} \mathrm{Cu}_{7.5} \mathrm{Ag}_{10}$ metallic glass, in which grains reach saturated values (about a few tens of nanometers) in a short time. Both Kashchiev's and Zeldovich's nucleation models (8 and 10) can fit well the experimental kinetic data. Furthermore, models 11 and 12, introducing a small time period $t_{o}$ in which grain grows to the saturated value, give similar fitting results as models 8 and 10. Figure 10 exemplifies the fitting curves with model 8 , showing how well such simple models (8 and 10-12) work at four temperatures in the annealing time range of $x$ $<0.7$. The derivations of the fits for relative volume fractions above 0.7 might be due to too simple growth models used here. The values of $\tau_{K}$ or $\tau_{Z}$ and $V_{o} I_{\mathrm{st}-K}$ or $V_{o} I_{\mathrm{st}-Z}$ obtained from the fitting using models 8 and 10 are listed in Table II. It is clearly seen that the incubation times deduced from Zeldovich's equation are nearly twice more than those obtained from the Kashchiev's equation, which is consistent with our previous observation in an Al-based amorphous alloy. ${ }^{35}$ Assuming an average grain size of $30 \mathrm{~nm}$, the steadstate nucleation rate $I_{\text {st }}$ can be estimated, e.g., $I_{\text {st }-K} \approx 1.6$

TABLE II. Parameters obtained from models 8 and 10.

\begin{tabular}{llcccc}
\hline & Models & $663 \mathrm{~K}$ & $673 \mathrm{~K}$ & $683 \mathrm{~K}$ & $693 \mathrm{~K}$ \\
\hline \multirow{2}{*}{ Model 8 } & $\tau_{K}(\mathrm{~min})$ & $80 \pm 5$ & $58 \pm 5$ & $38 \pm 3$ & $10 \pm 5$ \\
& $V_{0} I_{\mathrm{st}-K}\left(\mathrm{~min}^{-1}\right)$ & $0.014 \pm 0.002$ & $0.022 \pm 0.002$ & $0.025 \pm 0.005$ & $0.075 \pm 0.005$ \\
$E_{k}(\mathrm{~kJ} / \mathrm{mol})$ & & $253 \pm 45$ & & \\
& & \multicolumn{2}{c}{$100 \pm 5$} & $80 \pm 5$ & $20 \pm 5$ \\
\multirow{2}{*}{ Model 10 } & $\tau_{Z}(\mathrm{~min})$ & $155 \pm 5$ & $0.035 \pm 0.005$ & $0.058 \pm 0.005$ & $0.15 \pm 0.01$ \\
& $V_{0} I_{\mathrm{st}-Z}\left(\mathrm{~min}^{-1}\right)$ & $0.027 \pm 0.003$ & \multicolumn{2}{c}{$242 \pm 50$} \\
\hline \hline
\end{tabular}


$\times 10^{19} \mathrm{~m}^{-3} \mathrm{~s}^{-1}$ at $663 \mathrm{~K}, 2.6 \times 10^{19} \mathrm{~m}^{-3} \mathrm{~s}^{-1}$ at $673 \mathrm{~K}, 2.9$ $\times 10^{19} \mathrm{~m}^{-3} \mathrm{~s}^{-1}$ at $683 \mathrm{~K}$, and $8.8 \times 10^{19} \mathrm{~m}^{-3} \mathrm{~s}^{-1}$ at $693 \mathrm{~K}$. It seems that $I_{\mathrm{st}}$ increases with annealing temperature although the average grain sizes of quasicrystals might be slightly different at four temperatures. The steady-state nucleation rates obtained here are consistent with $3.8 \times 10^{20} \mathrm{~m}^{-3} \mathrm{~s}^{-1}$ at $700 \mathrm{~K}$ reported in the literature. ${ }^{13}$ The activation energy of nucleation for the amorphous-to-quasicrystalline phase transformation can be deduced from the plot of $\ln \tau$ with $1 / T$ and is also listed in Table II. It is found that the nucleation activation energy is similar to the effective activation energy deduced from the JMA model. The effective activation energy, deduced from the plot of $\ln k$ vs $1 / T$ in the JMA model, may correspond to both nucleation and growth processes, while it, deduced from the plot of $\ln \tau$ vs $1 / T$, is most likely linked with the nucleation process because during the initial stage of the crystallization (i.e., in the incubation time period), the nucleation process is dominant. This indicates that the amorphous-to-quasicrystalline phase transformation in the supercooled liquid region for the $\mathrm{Zr}_{65} \mathrm{Cu}_{7.5} \mathrm{Al}_{7.5} \mathrm{Ni}_{10} \mathrm{Ag}_{10}$ metallic glass may be mainly governed by the nucleation process, which is supported by the observation of high density of nucleation rate $I_{\text {st }}$ and nanometer-sized quasicrystalline grains in the $\mathrm{Zr}_{65} \mathrm{Cu}_{7.5} \mathrm{Al}_{7.5} \mathrm{Ni}_{10} \mathrm{Ag}_{10}$ alloys annealed in the supercooled liquid region.

In the study of the amorphous-to-quasicrystalline phase transformation, different mechanisms of the transformation were suggested. Knapp and Follstaedt ${ }^{36}$ explained the formation of quasicrystalline phase from amorphous alloys using the Stephens-Goldman model, ${ }^{37}$ in which the formation of the quasicrystalline phase is considered as packing small quasiunits while maintaining an orientational order. Lilienfeld et al. ${ }^{38}$ proposed that the transformation was due to either a nucleation and growth process or continuous transformation. A polymorphous transformation via a nucleation and growth process was suggested by Shen et al. ${ }^{39}$ in $\mathrm{Pd}-\mathrm{U}-\mathrm{Si}$ alloys and Holzer and Kelton ${ }^{24}$ in $\mathrm{Al}-\mathrm{Cu}-\mathrm{V}$ alloys while Chen et al. ${ }^{25}$ showed that the transformation in Al-Mn alloys occurs by a continuous growth process from quenched-in microquasicrystalline units with no nucleation step. In the amorphous-to-quasicrystalline phase transformation in the $\mathrm{Zr}_{65} \mathrm{Al}_{7.5} \mathrm{Ni}_{10} \mathrm{Cu}_{7.5} \mathrm{Ag}_{10}$ metallic glass, it is clear that the kinetic data at four temperatures obtained here cannot be described by models 1 and 2, ruling out a quenched-in "microquasicrystalline" unit model of the glass in the system studied. The transformation proceeds by time-dependent transient nucleation and growth. To further examine the contribution of the quenched-in nucleation to the transformation, models 13 and 14 were used to fit the experimental kinetic data. Good fits, as comparable to the models 8 and 10-12, can be achieved. It is noticed in Fig. 11 that in the region of low relative volume fractions, the fit introducing $2 \%\left(N V_{0}\right)$ quenched-in nuclei is even better than models 8 and 10-12. Assuming the average grain size of $30 \mathrm{~nm}$, the quenched-in nuclei number in the $\mathrm{Zr}_{65} \mathrm{Al}_{7.5} \mathrm{Ni}_{10} \mathrm{Cu}_{7.5} \mathrm{Ag}_{10}$ metallic glass is about $1.42 \times 10^{21} \mathrm{~m}^{-3}$.

\section{CONCLUSIONS}

The pressure effect on the formation of quasicrystals and the amorphous-to-quasicrystalline phase transformation

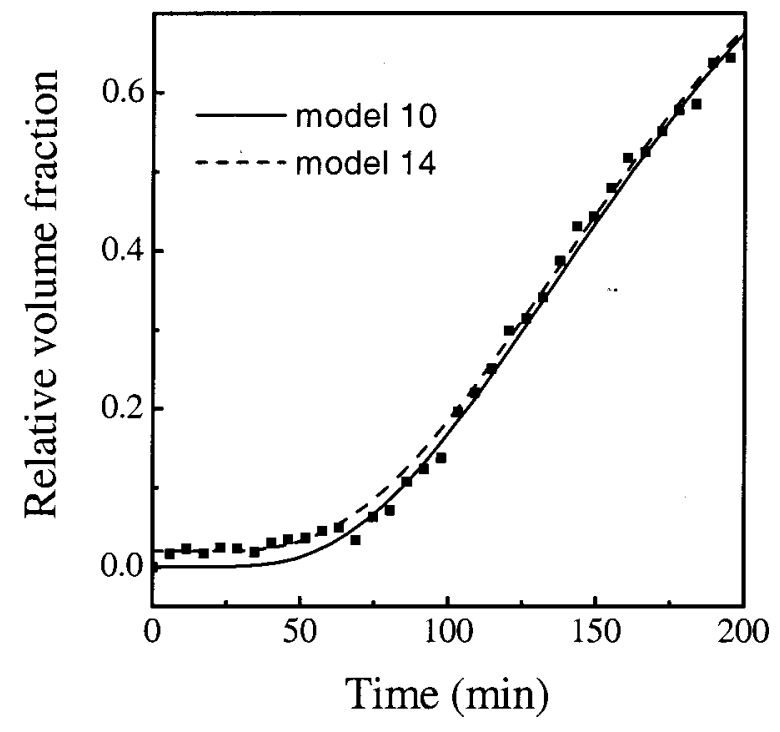

FIG. 11. Relative volume fractions of the quasicrystalline phase as a function of time during isothermal annealing treatments at 663 $\mathrm{K}$ and pressure of $0.86 \mathrm{GPa}$ with the fitting curves given by model 10 (solid line) and model 14 (dash line). The data (0-200 min) were selected to illustrate the differences in the fits for the incubation time and the fitting curves given by models 10 and 14 are almost the same after $200 \mathrm{~min}$.

kinetics in the supercooled liquid region for the $\mathrm{Zr}_{65} \mathrm{Cu}_{7.5} \mathrm{Al}_{7.5} \mathrm{Ni}_{10} \mathrm{Ag}_{10}$ metallic glass have been investigated by in situ high-pressure and high-temperature nonisothermal and isothermal energy-dispersive $\mathrm{x}$-ray powder diffraction measurements using synchrotron radiation, respectively. It is found that the external pressure enhances the onset temperature for the formation of quasicrystals with a rate of 9.4 $\mathrm{K} / \mathrm{GPa}$ while the temperature interval for the stability and the average grain size of quasicrystals decrease. The results, which could be explained by the suppression of atomic mobility under pressure, reveal that the formation of quasicrystals from the $\mathrm{Zr}_{65} \mathrm{Al}_{7.5} \mathrm{Ni}_{10} \mathrm{Cu}_{7.5} \mathrm{Ag}_{10}$ metallic glass may involve in atomic diffusion. To study the amorphous-toquasicrystalline phase transformation kinetics relative volume fractions of the quasicrystalline phase as a function of annealing time, obtained at 663, 673, 683, and $693 \mathrm{~K}$, have been analyzed in detail using 14 nucleation and growth models together with the JMA model. The Avrami exponent was found to be near 1 at all four temperatures, which also indicates an existence of atomic diffusion during the amorphous-to-quasicrystalline phase transformation in the metallic glass. The time-dependent transient nucleation, based on the Kashchiev's or Zeldovich's transient nucleation process, together with constant grain size can describe the experimental data obtained at four temperatures in the relative volume fraction of the quasicrystalline phase less than 70\%. The effective energy barrier for the amorphous-toquasicrystalline phase transformation was found to be approximately $230 \pm 60 \mathrm{~kJ} / \mathrm{mol}$. The steady-state nucleation rates are very high up to $10^{19} \mathrm{~m}^{-3} \mathrm{~s}^{-1}$ and increases with temperature. Quenched-in nucleation may exist in the glass with a relative volume fraction of $2 \%$. 


\section{ACKNOWLEDGMENTS}

We thank Hasylab in Hamburg, ESRF in Grenoble, and MAXLAB in Lund for use of the synchrotron radiation facilities. Financial support from the Danish Technical Research Council and the Danish Natural Sciences Research Council is gratefully acknowledged.

\section{APPENDIX}

The relative volume fraction $x(t)$ of the quasicrystalline phase during the amorphous-to-quasicrystalline phase transformation in the $\mathrm{Zr}_{65} \mathrm{Cu}_{7.5} \mathrm{Al}_{7.5} \mathrm{Ni}_{10} \mathrm{Ag}_{10}$ metallic glass as a function of time can be expressed as $x(t)=1-\exp [-Y(t)]$, where the expressions of $Y(t)$ for various nucleation and growth models are given as follows:

Model 1: quenched-in nucleation with three-dimensions constant growth rate $u$ :

$$
Y(t)=\frac{4}{3} N \pi u^{3} t^{3}
$$

where $N$ is the quenched-in nuclei number per unit volume, and $t$ is the annealing time.

Model 2: quenched-in nucleation with constant grain size $V_{0}$ :

$$
Y(t)=N^{*} V_{0} .
$$

Model 3: steady-state nucleation with three-dimensional constant growth rate:

$$
Y(t)=\int_{0}^{t} I_{\mathrm{st}} \frac{4}{3} \pi u^{3}(t-x)^{3} d x=\frac{1}{3} \pi I_{\mathrm{st}} u^{3} t^{4},
$$

where $I_{s t}$ is the steady-state nucleation rate.

Model 4: steady-state nucleation with constant grain size:

$$
Y(t)=\int_{0}^{t} I_{\mathrm{st}} V_{0} d x=I_{\mathrm{st}} V_{0} t
$$

Model 5: quenched-in and steady-state nucleation with threedimensional constant growth rate:

$$
\begin{aligned}
Y(t) & =\frac{4}{3} \pi N u^{3} t^{3}+\int_{0}^{t} I_{\mathrm{st}} \frac{4}{3} \pi u^{3}(t-x)^{3} d x \\
& =\frac{4}{3} \pi N u^{3} t^{3}+\frac{\pi}{3} I_{\mathrm{st}} u^{3} t^{4} .
\end{aligned}
$$

Model 6: quenched-in and steady-state nucleation with constant grain size:

$$
Y(t)=N V_{0}+\int_{0}^{t} I_{\mathrm{st}} V_{0} d x=N V_{0}+I_{\mathrm{st}} V_{0} t
$$

Model 7: time-dependent Kashchiev's nucleation $I(x)$ with three-dimensional constant growth rate:

$$
\begin{aligned}
Y(t)= & \int_{0}^{t} \frac{4}{3} \pi u^{3}(t-x)^{3} I(x) d x \\
= & \frac{4}{3} \pi I_{\mathrm{st}-K} u^{3}\left[\frac{t^{4}}{4}+2 \sum_{m=1}^{\infty}(-1)^{m} \int_{0}^{t} \exp \left(-\frac{m^{2} x}{\tau_{K}}\right)\right. \\
& \left.\times(t-x)^{3} d x\right]
\end{aligned}
$$

where $\tau_{K}$ and $I_{\text {st }-K}$ are the incubation time and the steadystate nucleation rate for the Kashchiev's nucleation, respectively.

Model 8: time-dependent Kashchiev's nucleation with constant grain size:

$$
\begin{aligned}
Y(t)= & \int_{0}^{t} I(x) V_{0} d x=I_{\mathrm{st}-K} V_{0} \\
& \times\left\{t+2 \sum_{m=1}^{\infty} \frac{(-1)^{m} \tau_{K}\left[1-\exp \left(-m^{2} t / \tau_{K}\right)\right]}{m^{2}}\right\} .
\end{aligned}
$$

Model 9: time-dependent Zeldovich's nucleation with threedimensional constant growth rate:

$$
\begin{aligned}
Y(t) & =\int_{0}^{t} \frac{4}{3} \pi u^{3}(t-x)^{3} I(x) d x \\
& =\frac{4}{3} \pi I_{\mathrm{st}-Z} u^{3} \int_{0}^{t} \exp \left(-\tau_{Z} / x\right)(t-x)^{3} d x,
\end{aligned}
$$

where $\tau_{Z}$ and $I_{\mathrm{st}-Z}$ are the incubation time and the steadystate nucleation rate for the Zeldovich's nucleation, respectively.

Model 10: time-dependent Zeldovich's nucleation with constant grain size:

$$
Y(t)=\int_{0}^{t} I(x) V_{0} d x=I_{\mathrm{st}-Z} V_{0} \int_{0}^{t} \exp \left(-\tau_{Z} / x\right) d x .
$$

Model 11: time-dependent Kashchiev's nucleation with three-dimensional constant growth rate at $t<t_{0}$ and zero growth rate at $t>t_{0}$ :

$$
Y(t)=\frac{4}{3} \pi I_{\mathrm{st}-K} u^{3}\left[\frac{t^{4}}{4}+2 \sum_{m=1}^{\infty}(-1)^{m} \int_{0}^{t} \exp \left(-\frac{m^{2} x}{\tau_{K}}\right)(t-x)^{3} d x\right]
$$

when $t \leqslant t_{0}$ and 


$$
\begin{aligned}
Y(t)= & \frac{4}{3} \pi I_{\mathrm{st}-K} u^{3}\left\{t_{0}^{3}\left[t-t_{0}+2 \sum_{m=1}^{\infty} \frac{(-1)^{m} \tau_{K}\left\{1-\exp \left[-m^{2}\left(t-t_{0}\right)\right] / \tau_{K}\right\}}{m^{2}}\right]\right. \\
& \left.+\left[\frac{t_{0}^{4}}{4}+2 \sum_{m=1}^{\infty}(-1)^{m} \int_{t-t_{0}}^{t} \exp \left(-\frac{m^{2} x}{\tau_{K}}\right)(t-x)^{3} d x\right]\right\} \\
= & \frac{4}{3} \pi I_{\mathrm{st}-K} u^{3}\left\{t_{0}^{3} t-\frac{3 t_{0}^{4}}{4}+2 \sum_{m=1}^{\infty}(-1)^{m}\left[\frac{\tau_{K} t_{0}^{3}\left[1-\exp \left(\frac{-m^{2}\left(t-t_{0}\right)}{\tau_{K}}\right)\right]}{m^{2}}+\int_{t-t_{0}}^{t} \exp \left(-\frac{m^{2} x}{\tau_{K}}\right)(t-x)^{3} d x\right)\right],
\end{aligned}
$$

when $t>t_{0}$

Model 12: time-dependent Zeldovich's nucleation with three-dimensional constant growth rate at $t<t_{0}$ and zero growth rate at $t>t_{0}$ :

$$
Y(t)=\frac{4}{3} \pi I_{\mathrm{st}-Z} u^{3} \int_{0}^{t} \exp \left(-\tau_{Z} / x\right)(t-x)^{3} d x,
$$

when $t \leqslant t_{0}$ and

$$
\begin{aligned}
Y(t)= & \frac{4}{3} \pi u^{3} I_{\mathrm{st}-Z}\left[t_{0}^{3} \int_{0}^{t-t_{0}} \exp \left(-\tau_{Z} / x\right) d x\right. \\
& \left.+\int_{t-t_{0}}^{t} \exp \left(\tau_{Z} / x\right)(t-x)^{3} d x\right], \text { when } t>t_{0} .
\end{aligned}
$$

Model 13: quenched-in and time-dependent Zeldovich's nucleation with three-dimensional constant growth rate:

$$
Y(t)=\frac{4}{3} N \pi u^{3} t^{3}+\frac{4}{3} \pi I_{\mathrm{st}-Z} u^{3} \int_{0}^{t} \exp \left(-\tau_{Z} / x\right)(t-x)^{3} d x
$$

Model 14: quenched-in and time-dependent Zeldovich's nucleation with constant grain size:

$$
Y(t)=N V_{0}+I_{\mathrm{st}-Z} V_{0} \int_{0}^{t} \exp \left(-\tau_{Z} / x\right) d x .
$$

*Corresponding author. Electronic address: jiang@fysik.dtu.dk

${ }^{1}$ U. Koster, J. Meinhardt, S. Roos, and H. Liebertz, Appl. Phys. Lett. 69, 179 (1996); U. Koster, J. Meinhardt, S. Roos, and A. Rudiger, Mater. Sci. Forum 225, 311 (1996).

${ }^{2}$ J. Saida, M. Matsushita, and A. Inoue, Appl. Phys. Lett. 77, 73 (2000).

${ }^{3}$ B. S. Murty, D. H. Ping, and K. Hono, Appl. Phys. Lett. 77, 1102 (2000).

${ }^{4}$ J. Saida, M. Matsushita, C. Li, and A. Inoue, Appl. Phys. Lett. 76, 3558 (2000).

${ }^{5}$ S. Yi and D. H. Kim, J. Mater. Res. 15, 892 (2000).

${ }^{6}$ B. S. Murty, D. H. Ping, K. Hono, and A. Inoue, Appl. Phys. Lett. 76, 55 (2000).

${ }^{7}$ B. S. Murty, D. H. Ping, K. Hono, and A. Inoue, Scr. Mater. 43, 103 (2000).

${ }^{8}$ U. Koster, J. Meinhardt, S. Roos, and R. Busch, Mater. Sci. Eng., A 226, 995 (1997); D. Zander, R. Janlewing, A. Rudiger, and U. Koster, Mater. Sci. Forum 307, 25 (1999).

${ }^{9}$ A. Inoue, J. Saida, M. Matsushita, and T. Sakurai, Mater. Trans., JIM 41, 362 (2000).

${ }^{10}$ J. Eckert, N. Mattern, M. Zinkevitch, and M. Seidel, Mater. Trans., JIM 39, 623 (1998).

${ }^{11}$ L. Q. Xing, J. Eckert, W. Loser, and L. Schultz, Appl. Phys. Lett. 73, 2110 (1998); 74, 664 (1999).

${ }^{12}$ M. W. Chen, T. Zhang, A. Inoue, A. Sakai, and T. Sakural, Appl. Phys. Lett. 75, 1697 (1999); M. W. Chen, A. Inoue, T. Zhang, A. Sakai, and T. Sakurai, Intermetallics 8, 493 (2000); Philos. Mag. Lett. 80, 263 (2000).

${ }^{13}$ A. Inoue, T. Zhang, J. Saida, M. Matsushita, M. W. Chen, and T.
Sakural, Mater. Trans., JIM 40, 1137 (1999); 40, 1181 (1999); A. Inoue, T. Zhang, M. W. Chen, and T. Sakural, ibid. 40, 1382 (1999); A. Inoue, T. Zhang, M. W. Chen, T. Sakurai, J. Saida, and M. Matsushita, J. Mater. Res. 15, 2195 (2000).

${ }^{14}$ J. Saida, M. Matsushita, T. Zhang, A. Inoue, M. W. Chen, and T. Sakural, Appl. Phys. Lett. 75, 3497 (1999); M. Matsushita, J. Saida, T. Zhang, A. Inoue, M. W. Chen, and T. Sakural, Philos. Mag. Lett. 80, 79 (2000); J. Saida, M. Matsushita, C. Li, and A. Inoue, ibid. 80, 737 (2000).

${ }^{15}$ A. Inoue, T. Zhang, M. W. Chen, T. Sakurai, J. Saida, and M. Matsushita, Appl. Phys. Lett. 76, 967 (2000).

${ }^{16}$ J. K. Lee, G. Choi, D. H. Kim, and W. T. Kim, Appl. Phys. Lett. 77, 978 (2000).

${ }^{17}$ N. Wanderka, M.-P. Macht, M. Seidel, S. Mechler, K. Ståhl, and J. Z. Jiang, Appl. Phys. Lett. 77, 3935 (2000).

${ }^{18}$ J. Z. Jiang, J. S. Olsen, L. Gerward, S. Abdali, J. Eckert, N. Schlorke-de Boer, L. Schultz, J. Truckenbrodt, and P. X. Shi, J. Appl. Phys. 87, 2664 (2000).

${ }^{19}$ J. Z. Jiang, T. J. Zhou, H. K. Rasmussen, U. Kuhn, J. Eckert, and C. Lathe, Appl. Phys. Lett. 77, 3553 (2000).

${ }^{20}$ Y. X. Zhuang, J. Z. Jiang, T. J. Zhou, H. Rasmussen, L. Gerward, M. Mezouar, W. Crichton, and A. Inoue, Appl. Phys. Lett. 77, 4133 (2000).

${ }^{21}$ J. Z. Jiang, Y. X. Zhuang, H. Rasmussen, N. Nishiyama, A. Inoue, and C. Lathe, Europhys. Lett. 54, 182 (2001).

${ }^{22}$ J. S. Olsen, L. Gerward, and J. Z. Jiang, J. Phys. Chem. Solids 60, 229 (1999).

${ }^{23}$ D. L. Decker, J. Appl. Phys. 42, 3239 (1971).

${ }^{24}$ J. C. Holzer and K. F. Kelton, Acta Metall. Mater. 39, 1833 (1991). 
${ }^{25}$ C. L. Chen, F. Spaepen, J. L. Robertson, S. C. Moss, and K. Hiraga, J. Mater. Res. 5, 1871 (1990).

${ }^{26}$ A. Inoue, D. Kawase, A. P. Tsai, and T. Zhang, and T. Masumoto, Mater. Sci. Eng., A 178, 255 (1994).

${ }^{27}$ M. Sutton, Y. S. Yang, J. Mainville, J. L. Jordan-Sweet, K. F. Ludwig, and G. B. Stephenson, Phys. Rev. Lett. 62, 288 (1989); S. Brauer, J. O. Strom-Olsen, M. Sutton, Y. S. Yang, and A. Zaluska, G. B. Stephenson, and U. Koster, Phys. Rev. B 45, 7704 (1992); S. Brauer, H. E. Fischer, J. O. Strom-Olsen, M. Sutton, A. Zaluska, and G. B. Stephenson, ibid. 47, 11757 (1993).

${ }^{28}$ W. A. Johnson and R. F. Mehl, Trans. Am. Inst. Min., Metall. Pet. Eng. 135, 416 (1939).

${ }^{29}$ M. Avrami, J. Chem. Phys. 9, 177 (1941).

${ }^{30}$ H. Yinnon and D. R. Uhlmann, J. Non-Cryst. Solids 54, 253 (1983).

${ }^{31}$ D. Kashchiev, Surf. Sci. 14, 209 (1969).
${ }^{32}$ J. B. Zeldovich, Acta Physicochim. URSS 18, 1 (1943); J. I. Frenckel, Kinetic Theory of Liquids (Oxford University Press, London, 1946).

${ }^{33}$ M. Bushwitz, R. Adlwarth-Dieball, and P. L. Ryder, Acta Metall. Mater. 41, 1885 (1993).

${ }^{34}$ I. Gutzow, Contemp. Phys. 21, 121 (1980).

${ }^{35}$ Y. X. Zhuang, J. Z. Jiang, Z. G. Lin, M. Mezouar, W. Crichton, and A. Inoue, Appl. Phys. Lett. 79, 743 (2001).

${ }^{36}$ J. A. Knapp and D. M. Follstaedt, Phys. Rev. Lett. 55, 1591 (1985).

${ }^{37}$ P. W. Stephens and A. I. Goldman, Phys. Rev. Lett. 56, 1168 (1986).

${ }^{38}$ D. A. Lilienfeld, M. Nastasi, H. H. Johnson, D. G. Ast, and J. W. Mayer, Phys. Rev. Lett. 55, 1587 (1985).

${ }^{39}$ S. J. Poon, A. J. Drehman, and K. R. Lawless, Phys. Rev. Lett. 55, 2324 (1985); Y. Shen, S. J. Poon, and G. J. Shiflet, Phys. Rev. B 34, 3516 (1986). 